\title{
Electrostatic end-field defocusing of neutral atoms and its compensation
}

\author{
Juris G. Kalnins \\ Lawrence Berkeley National Laboratory, MS-71R0211, Berkeley, California 94720, USA
}

(Received 23 February 2011; published 11 October 2011)

\begin{abstract}
Neutral atoms entering an electric field experience a defocusing force in the dipole field direction, which is proportional to the field gradient. If an experiment, such as the search for a permanent electron electric dipole moment (eEDM), requires a very strong electric field $(13.5 \mathrm{MV} / \mathrm{m})$, then this end-field defocusing results in beam blowup and much reduced phase-space acceptance. In this paper we discuss how these defocusing fields arise from the longitudinal changes in the electric dipole field and their dependence on the electrode shape and spacing between lenses. We find that the end-field defocusing comes from strong impulse forces, whose defocusing power was calculated for simple electrodes with rounded ends. To compensate for this end-field defocusing, a triplet of transverse-focusing lenses was added to the pure dipole field plates in the generic eEDM cesium fountain experiment used to study the neutral beam optics. Envelope equations, which calculated the beam sizes of the atom bunch for the linear forces, are used to obtain a set of lens parameters that give a well focused beam in the fountain. Atom trajectory equations allow us to calculate the phase-space acceptance of the lens system with the nonlinear force terms included.
\end{abstract}

DOI: 10.1103/PhysRevSTAB.14.104201

PACS numbers: 41.85.Ja, 41.85.Lc, 41.85.Ne

\section{INTRODUCTION}

A beam of slow neutral atoms in a static electric field gradient experiences a force arising from the interaction of the electric field with the induced electric dipole moment of the atom, which can produce useful focusing [1] as well as undesirable end-field defocusing.

Cesium atoms have a polarizability such that it produces forces that are in the direction of the strongest electric field (strong field seeking). Therefore the atoms on entering an electric dipole field will be accelerated in the longitudinal direction by the rising field strength, and then decelerated when they leave the electric field region (Sec. VI). In addition, at the entrance (and exit) to the electrodes the electric field strength also increases off axis in the electric field direction (Fig. 1), so the atoms will experience an end-field defocusing force (Sec. IV) at both ends. Inside the dipole field, where the field strength is essentially constant, there will be no forces on the atom and it will act just like a drift space. If we add a transverse sextupole field to the dipole field of the lens (Sec. V), then inside the plates the electric field will increase off axis in one transverse direction and decrease in the other-producing the same type of focusing as quadrupole magnets on charged particles.

In previous publications describing the use of static inhomogeneous electric fields to focus (strong-fieldseeking) neutral atoms and molecules, end effects were

Published by the American Physical Society under the terms of the Creative Commons Attribution 3.0 License. Further distribution of this work must maintain attribution to the author(s) and the published article's title, journal citation, and DOI. neglected [2-5]. This was justified because the lens length was long enough, the transverse electric field gradient large enough, and the electric dipole field small enough that the lens focusing/defocusing forces were large compared to the end-field effects. In addition, some sets of focusing lenses were designed to operate with each element at nearly the same voltage and with only small separations between elements, so that there were no significant end

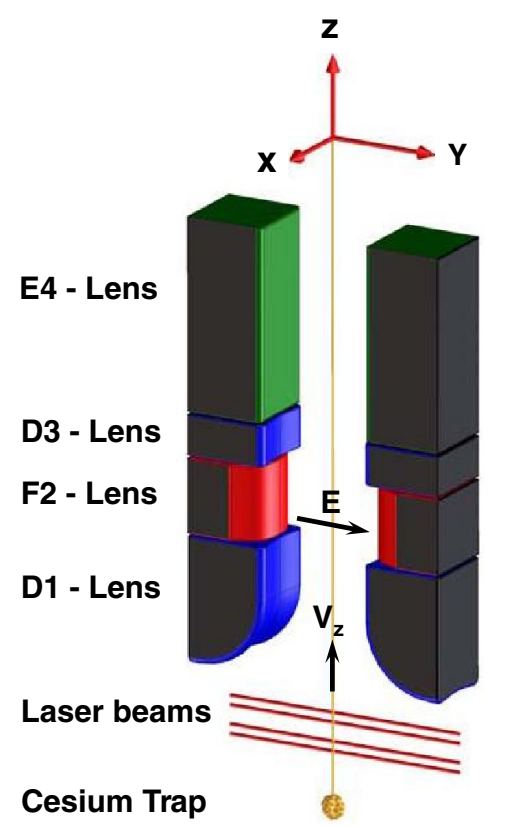

FIG. 1. Schematic of the electrostatic lenses in the generic Cs fountain. The D1 and D3 are concave $y$-focusing/ $x$-defocusing lenses, F2 is a convex $x$-focusing/y-defocusing lens, and E4 is a nonfocusing pure dipole field lens. 


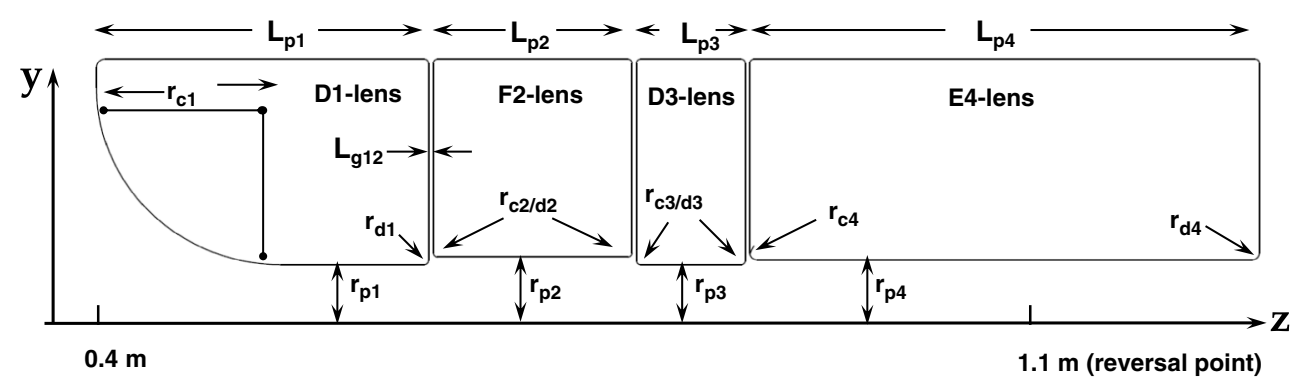

FIG. 2. The $(y, Z)$ cross section of the electrodes in the Cs fountain lenses. The electrode ends are rounded off with $1 / 4$ circles of radii $r_{c}, r_{d}$.

effects between the lens elements (Sec. IV B). The effectiveness of this approach was demonstrated by focusing a beam of slow neutral cesium atoms with an electrostatic triplet [5].

In experiments with thermal or supersonic beams traveling 2 orders of magnitude faster than considered here, the beam rigidity is so large that the end-field effects produce much less defocusing (see for example [6]).

However, in experiments using slow atoms with high polarizabilities in strong electric fields, the beam will defocus strongly in the $y$ (field) direction (Fig. 1) on entering and leaving the electric dipole field, resulting in a much larger beam size.

In Sec. IV, we will show that (for electrodes rounded off at the ends by quarter circles) in the lens end field, or the gap between lenses, we have one or more short-range impulse forces both focusing and defocusing, though in general with net defocusing. We find the change in an atom's transverse $v_{y}$ velocity produced by one of the impulse fields, for the linear part of the force, is $\Delta v_{y}=\frac{\alpha}{m}\left(V_{p}^{2} / V_{z}\right)\left(p_{y 2} / r_{p}^{3}\right) y$, where $\alpha$ is the ground-state dipole polarizability, $m$ is the mass, $V_{z}$ is the total longitudinal velocity, $y$ is the displacement off axis, $V_{p}$ is the plate potential, $r_{p}$ is the plate half gap, and $p_{y 2}$ is a dimensionless linear-force defocusing power $\left(p_{y 2}>0\right)$ that depends on the shape of the plate ends (Fig. 2, Sec. IV). So to compensate for the end-field defocusing, besides a low plate potential $V_{p}$, we want electrodes with the largest half gap $r_{p}$ and shaped ends which give the smallest defocusing power $p_{y 2}$.

For the cesium fountain considered here, the end-field defocusing by a $13.5 \mathrm{MV} / \mathrm{m}$ dipole field reduced the phase-space acceptance area by a factor of 2000, from that of the maximum geometric acceptance (Sec. IVA). This was due to the large increase in the $y$ velocity produced by the entrance and exit defocusing fields-for an atom entering the lens with longitudinal velocity $V_{z}=3.0 \mathrm{~m} / \mathrm{s}$ and displacement $y=2.5 \mathrm{~mm}$, the velocity change is $\Delta v_{y}=155 \mathrm{~mm} / \mathrm{s} \quad\left(V_{p}=67.5 \mathrm{kV}, \quad r_{p}=5 \mathrm{~mm}, p_{y 2}=\right.$ 0.17 ), which is very large compared with the $8 \mathrm{~mm} / \mathrm{s} \mathrm{rms}$ thermal velocity. So a set of additional transverse-focusing lenses are needed to control the transverse bunch size and maximize the phase-space acceptance.
Examples of experiments using slow atoms in uniform electric fields include: a measurement of the cesium hyperfine splitting Stark shift, used to correct for black body radiation effects in atomic clocks [7]; the cesium static polarizability [8]; and a cesium fountain experiment to search for a permanent electric dipole moment (eEDM) of the electron [9]. However, the hyperfine splitting Stark shift experiment and the static polarizability experiment are precision measurements requiring a detailed knowledge of the electric field along the trajectory of the atoms. This may not be compatible with the use of transversefocusing lenses, which introduce additional variations in the electric field to that already in the longitudinal direction. Without focusing lenses the optimization of the shape of the dipole field end becomes critically important for future measurements of these types. The electron EDM experiment however can use transverse-focusing lenses because it only looks for differences in the atom's energy levels when its spin is aligned and antialigned with the electric field.

In this paper we will investigate the focusing properties of electrostatic lenses in the context of a generic electron electric dipole moment (eEDM) experiment cesium fountain (Sec. II). We discuss electric field-gradient focusing for strong-field-seeking atoms in Sec. III, and the two types of electric fields produced by lenses in $\operatorname{Sec}$. IV $[(y, z)$ longitudinal] and Sec. V $[(x, y)$ transverse $]$. For these fields we present the trajectory equations of motion in Sec. VI and the beam envelope equations in Sec. VII (for the forces that are linear with the atom displacement off axis). Conclusions are drawn in Sec. VIII.

\section{II. eEDM FOUNTAIN}

The generic electron electric dipole moment experiment cesium fountain is shown in Fig. 1 and consists of three transverse-focusing lenses (D1, F2, D3 triplet) followed by pure dipole electric field plates (E4) (called "lenses" even though they do not focus), with all the dipole fields in the $y$ direction. To minimize field breakdown in the small longitudinal gaps between the lenses, all of the pairs of lens electrodes are set to the same potential. With gaps between the lenses, we can use separate power supplies to 
TABLE I. Lens parameters: distance to first lens $(\mathrm{D} 1)=0.40 \mathrm{~m}$ : lens gap spacing $L_{g}=1.5 \mathrm{~mm}$ : plate potential $V_{p}=67.5 \mathrm{kV}$.

\begin{tabular}{lcccccc}
\hline \hline Lens & $\begin{array}{c}\text { Plate length } \\
L_{p}(\mathrm{~m})\end{array}$ & $\begin{array}{c}\text { Plate half } \\
\text { gap } r_{p}(\mathrm{~mm})\end{array}$ & $\begin{array}{c}\text { Corner radius } \\
r_{c}(\mathrm{~mm})\end{array}$ & $\begin{array}{c}\text { Corner radius } \\
r_{d}(\mathrm{~mm})\end{array}$ & $\begin{array}{c}\text { Electric field } \\
E_{p}(\mathrm{MV} / \mathrm{m})\end{array}$ & $\begin{array}{c}\text { Sextupole strength } \\
A_{3}\left(\mathrm{~m}^{-2}\right)\end{array}$ \\
\hline D1 & 0.1135 & 6 & 60 & 1.5 & 11.3 & +400 \\
F2 & 0.0669 & 6 & 1.5 & 1.5 & 11.2 & -400 \\
D3 & 0.0362 & 6 & 1.5 & 1.5 & 1.3 & +400 \\
E4 & 0.70 & 5 & 2.5 & 2.5 & 13.5 & 0 \\
\hline \hline
\end{tabular}

apply slightly different voltages and fine-tune the triplet focusing (as was done in $[1,5]$ ).

The electrode shapes of the lenses in the $(y, Z)$ plane are shown in Fig. 2, with the plate ends rounded off with quarter circles of radii $r_{c}$ and $r_{d}$. The electrostatic potential and fields of these lenses was calculated from Laplace's equation using the program flexPDE ${ }^{\mathrm{TM}}$ (PDE Solutions Inc.).

The cesium bunch in the generic trap has a temperature of $0.5 \mu \mathrm{K}$ and each bunch is launched with a velocity of $4.0 \mathrm{~m} / \mathrm{s}$, emptying the trap $[10,11]$. The initial atom distribution has an rms size of $4.5 \mathrm{~mm}$ and rms relative velocity of $8 \mathrm{~mm} / \mathrm{s}$. The distance from the trap to the trajectory reversal point is $1.10 \mathrm{~m}$ with a transit time of $0.50 \mathrm{~s}$.

The main physical parameters of the four lenses are given in Table I.

The eEDM experiment requires the $\mathrm{E} 4$ lens to have a very large electric field of $E_{p}=13.5 \mathrm{MV} / \mathrm{m}$. To maximize the phase-space acceptance of the atom bunch in the $y$ direction, the plate half gap $r_{p}$ should be as large as possible. Choosing a maximum plate potential of $V_{p}=$ $67.5 \mathrm{kV}$ gives us a half gap of $r_{p}=5 \mathrm{~mm}$.

In the transverse $(x, y)$ plane, the (D1, F2, D3)-lens dipole-sextupole field linear forces, each focus the atom in one direction while defocusing equally in the other (the same as a quadrupole lens in charged particle optics). The $F$ lenses (convex: $A_{3}<0$ ) focus in the $x$ direction and defocus in the $y$ direction, while the $\mathrm{D}$ lenses (concave: $A_{3}>0$ ) defocus in the $x$ direction and focus in the $y$ direction. Combining two or more of these focusing lenses (e.g. doublets FD, DF or triplets FDF, DFD) will produce net focusing in both $x$ and $y$ directions, and in beam optics this is known as alternating gradient focusing. Because of the very strong end-field defocusing of the atom bunch in the dipole field direction on entering the first lens (D1), it was chosen to be focusing in the $y$ direction (see Fig. 1). Triplet lenses with the DFD configuration produce focusing that gives more control and the smallest beam size in the $y$ direction, where the phasespace acceptance is strongly limited by the physical plate apertures and the end-field defocusing. To minimize the aberrations from the nonlinear forces in the (D1, F2, D3) lenses [see Sec. VI, Eq. (10)] we chose a small sextupole field strength $\left|A_{3}\right|=400 \mathrm{~m}^{-2}$ (with $r_{p}^{2}\left|A_{3}\right| \ll 1$ ) for the three lenses.
The eEDM-fountain design optics (Table I: triplet lengths $L_{p}$ ) were obtained using the linear-focusing beam envelope equations (see Sec. VII), and varying the (D1, F2, D3)-lens plate lengths $L_{p}$ to change the transverse focusing, and obtain the smallest beam sizes in the fountain with maximum phase-space acceptance areas.

\section{ELECTRIC FIELD-GRADIENT FOCUSING}

The Hamiltonian for a neutral cesium atom in a fountain with electric fields, in the coordinate system of Fig. 1, is

$$
H=1 / 2 m v^{2}+m g Z+W,
$$

where $v=\sqrt{v_{x}^{2}+v_{y}^{2}+V_{z}^{2}}$ is the total velocity, $m=$ $2.207 \times 10^{-25} \mathrm{~kg}$ is the cesium mass, $g=9.80 \mathrm{~ms}^{-2}$ is the acceleration of gravity, and

$$
W=-1 / 2 \alpha E^{2}
$$

is the potential energy of the ground-state atom in an electric field of magnitude $E=\sqrt{E_{x}^{2}+E_{y}^{2}+E_{z}^{2}}$ and $\alpha=$ $(6.61 \pm 0.009) \times 10^{-39} \mathrm{Jm}^{2} \mathrm{~V}^{-2}$ is the cesium static dipole polarizability.

The force on the atom due to the electric field is then

$$
\mathbf{F}=-\nabla W=\alpha E \nabla E
$$

and is directed towards the stronger field regions (strongfield seeking) but independent of the direction of the field.

Since all the electric fields in the eEDM-fountain lenses are static, the Hamiltonian energy [Eq. (1) and (2)] is conserved. Therefore on entering the electric field of a lens an atom will be accelerated [Eq. (3)] to a higher kinetic energy and it will lose kinetic energy on leaving.

The equations of motion of an atom in the fountain, for the Hamiltonian of Eqs. (1) and (2), are

$$
\begin{aligned}
& \frac{d v_{x}}{d t}=-\frac{1}{m} \frac{\partial H}{\partial x}=\frac{\alpha}{2 m} \frac{\partial E^{2}}{\partial x} \\
& \frac{d v_{y}}{d t}=-\frac{1}{m} \frac{\partial H}{\partial y}=\frac{\alpha}{2 m} \frac{\partial E^{2}}{\partial y} \\
& \frac{d V_{z}}{d t}=-\frac{1}{m} \frac{\partial H}{\partial Z}=-g+\frac{\alpha}{2 m} \frac{\partial E^{2}}{\partial Z},
\end{aligned}
$$


where we integrate the trajectories in time $t$, rather than the distance $Z$, since the longitudinal velocity $V_{z}$ goes to zero at the turnaround point.

The atom bunch has a central trajectory which has the average phase-space coordinates $\langle x\rangle=0,\left\langle v_{x}\right\rangle=0$, $\langle y\rangle=0,\left\langle v_{y}\right\rangle=0,\langle Z\rangle=Z_{c},\left\langle V_{z}\right\rangle=V_{z c}$ and ideally moves along the optic axis of the lenses. Any single atom in the bunch is then specified by the relative $6 \mathrm{D}$ phase-space coordinates $\left(x, v_{x}, y, v_{y}, z=Z-Z_{c}, v_{z}=V_{z}-V_{z c}\right)$.

If the forces in the $(x, y, z)$ directions are uncoupled, then we can look at the optics in the three independent 2D phase spaces $\left(x, v_{x}\right)$, etc. with the atom distribution specified by the rms moments $\sigma_{x}^{2}=\left\langle x^{2}\right\rangle, \sigma_{x v_{x}}=\left\langle x v_{x}\right\rangle, \sigma_{v_{x}}^{2}=\left\langle v_{x}^{2}\right\rangle$, etc. The atom bunch trajectories in phase space occupy an ellipse of emittance area $A_{x}=\pi \varepsilon_{x}=$ $\pi \sqrt{\sigma_{x}^{2} \sigma_{v_{x}}^{2}-\sigma_{x v_{x}}^{2}}$, etc.

If the forces on the atom are linear with the displacement off axis $\left(F_{x} \propto x\right.$, etc. $)$ then the phase-space emittance area is conserved. From charged particle optics, we know that the nonlinear terms in the force result in emittance growth, larger bunch size and relative velocity, with reduced atom density and beam loss from the outer edges of the bunch. Therefore it is important to design lenses with electric fields that produce forces with only a small nonlinear contribution.

For a given set of lens fields (such as given in Table I), we can calculate the initial phase-space atom trajectories in the fountain and determine which ones will be transported to the final target/detector without being lost to lens apertures, detector sizes, nonlinear forces, etc. The acceptance of the fountain is then the $6 \mathrm{D}$ phase-space volume (or 2D areas) occupied by the transmitted trajectories. Thus the number of atoms transmitted to the detector will be given by the number of atoms of the initial distribution that lie inside the phase-space acceptance volume. In Sec. IV we calculate the acceptance area for the $\left(y, v_{y}\right)$ phase space, where the end-field defocusing occurs.

In the eEDM fountain the electric fields that are produced by the lenses are of two types: (1) longitudinal gradient fields from primarily $(y, Z)$ potentials and (2) transverse gradient fields from primarily $(x, y)$ potentials. The characteristics of these electric fields and their effects on the atom motion are presented in the next two sections.

\section{EFFECTS OF LENS END FIELDS ON THE BEAM}

We look here at the electric dipole field and its gradient in the longitudinal $Z$ direction due to the electrode shape and proximity to other lenses in the $(y, Z)$ plane, as shown in Fig. 2. For pure dipole field lenses, with electrodes that are flat in the $x$ direction $(\partial \Phi / \partial x=0)$, the electrostatic potential $\Phi(y, Z)$ in an off-axis expansion is
$-\Phi(y, Z)=E_{y o}(Z) y-\frac{1}{3 !} E_{y 2}(Z) y^{3}+\frac{1}{5 !} E_{y 4}(Z) y^{5}+\cdots$,

where $E_{y o}(Z) \equiv E(y=0, Z)$ is the field on axis, with derivatives $E_{y 1} \equiv d E_{y o} / d Z$ etc.

The total electric field can then be written in the form

$$
\begin{aligned}
E^{2}(y, Z)= & E_{y o}^{2}(Z)+D_{y 2}(Z) y^{2}+\frac{1}{2} D_{y 4}(Z) y^{4} \\
& +\frac{1}{3} D_{y 6}(Z) y^{6}+\cdots,
\end{aligned}
$$

where the longitudinal field functions $D_{y 2}, D_{y 4}$, etc. can be calculated from the field on axis

$D_{y 2}=E_{y 1}^{2}-E_{y o} E_{y 2}$

$D_{y 4}=\frac{1}{2} E_{y 2}^{2}-\frac{2}{3} E_{y 1} E_{y 3}+\frac{1}{6} E_{y o} E_{y 4}$

$D_{y 6}=\frac{1}{12} E_{y 3}^{2}-\frac{1}{8} E_{y 2} E_{y 4}+\frac{1}{20} E_{y 1} E_{y 5}-\frac{1}{120} E_{y o} E_{y 6}$.

In general there are higher order terms in the total electric field, but for the lenses considered here, they were found to be negligible.

The longitudinal electric field [Eq. (4)] produces the acceleration in the $y$ direction

$$
\frac{d v_{y}}{d t}=\frac{\alpha}{m} D_{y e}(y, Z) y,
$$

where $\quad D_{y e}(y, Z) \equiv \frac{1}{2 y} \frac{\partial E^{2}}{\partial y}=D_{y 2}(Z)+D_{y 4}(Z) y^{2}+D_{y 6}(Z) y^{4}$ and the acceleration will be defocusing if $D_{y e}>0$.

To see the dependence of the acceleration on the electrode half gap $r_{p}$ and the maximum dipole field $E_{p}=$ $V_{p} / r_{p}$ for plate potentials $\pm V_{p}$, we use the dimensionless form of the $D_{y e}$ field function: $d_{y e}\left(\frac{y}{r_{p}}, \frac{Z}{r_{p}}\right) \equiv\left[r_{p}^{2} /\left(2 E_{p}^{2} y\right)\right] \times$ $\frac{\partial E^{2}}{\partial y}=d_{y 2}\left(\frac{Z}{r_{p}}\right)+d_{y 4}\left(\frac{Z}{r_{p}}\right)\left(y^{2} / r_{p}^{2}\right)+d_{y 6}\left(\frac{Z}{r_{p}}\right)\left(y^{4} / r_{p}^{4}\right)$ for which the acceleration becomes $\frac{d v_{y}}{d t}=\frac{\alpha}{m}\left(V_{p}^{2} / r_{p}^{4}\right) d_{y e}\left(\frac{y}{r_{p}}, \frac{Z}{r_{p}}\right) y$

For the eEDM fountain, the electric field and its first two derivatives on axis are shown in Fig. 3 and the resulting dimensionless linear-focusing field $d_{y 2}$ [calculated from Eq. (5)] is plotted in Fig. 4 (normalized with respect to the E4-lens half gap $r_{p}=5 \mathrm{~mm}$ and electric field $E_{p}=$ $13.5 \mathrm{MV} / \mathrm{m}$ ). It consists of sets of short-range focusing $\left(d_{y 2}<0\right)$ and defocusing $\left(d_{y 2}>0\right)$ peaks at the entrance to the first lens and in the gaps between the lenses.

At each peak, in the impulse approximation (with displacement off axis: $y_{f}=y_{i}$ ), we can calculate the change in transverse velocity ( $i=$ initial, $f=$ final)

$$
v_{y f}=v_{y i}+\frac{\alpha}{m} \frac{V_{p}^{2}}{V_{z i f}} \frac{y_{i}}{r_{p}^{3}}\left[p_{y 2}+p_{y 4} \frac{y_{i}^{2}}{r_{p}^{2}}+p_{y 6} \frac{y_{i}^{4}}{r_{p}^{4}}\right],
$$

where $V_{z i f}$ is the average longitudinal velocity and the dimensionless focusing/defocusing powers $p_{y 2}, p_{y 4}, p_{y 6}$ are given by the dimensionless integrals over the peak 


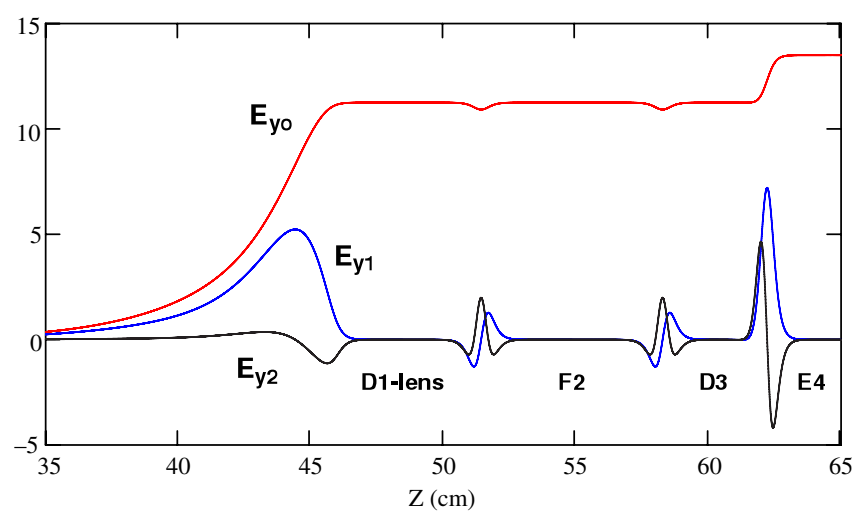

FIG. 3. The electric field on axis $E_{y o}(\mathrm{MV} / \mathrm{m})$ and its first two derivatives $E_{y 1}\left(2 \times 10^{-2} \mathrm{MV} / \mathrm{m}^{2}\right), E_{y 2}\left(5 \times 10^{-5} \mathrm{MV} / \mathrm{m}^{3}\right)$ in the Cs fountain.

$$
\begin{gathered}
p_{y 2}=\int d_{y 2}\left(\frac{Z}{r_{p}}\right) \frac{d Z}{r_{p}} \quad p_{y 4}=\int d_{y 4}\left(\frac{Z}{r_{p}}\right) \frac{d Z}{r_{p}} \\
p_{y 6}=\int d_{y 6}\left(\frac{Z}{r_{p}}\right) \frac{d Z}{r_{p}}
\end{gathered}
$$

and whose values are determined by the physical shape and spacing of the electrodes.

Equation (6) shows us that, to reduce the end-field defocusing (velocity change), we lower the plate potential and/or increase the plate gap size. However, for the eEDM experiment we need a strong electric field of $13.5 \mathrm{MV} / \mathrm{m}$ in the $\mathrm{E} 4$ lens, so for large plate gaps $(5 \mathrm{~mm})$ this requires large electrode potentials $(67.5 \mathrm{kV})$ whose maximum value will be limited by possible electric field breakdown.

The end fields that we encounter in the eEDM fountain are of two types - the field at the entrance of the lenses (Fig. 2: D1) and those in the gaps between the lenses (Fig. 2: D1-F2, F2-D3, D3-E4). In the eEDM-fountain electrode design, the physical parameters (Table I) were chosen to minimize the net defocusing from these four end-field regions.

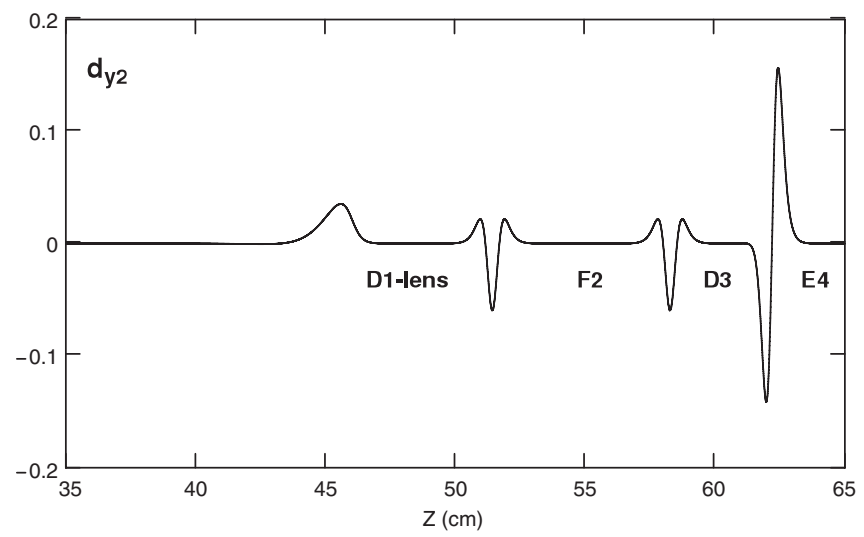

FIG. 4. The acceleration of the atom in the $y$ direction is proportional to the dimensionless end-field focusing/defocusing field $d_{y 2}$ (the linear term), and is shown here for the four lenses in the Cs fountain.

\section{A. End field at the entrance/exit of a single lens}

The greatest change in the electric field usually occurs when the atom bunch enters a lens from a field-free region (or the reverse) which typically produces the most defocusing of the atoms in the dipole field $y$ direction.

The lens in the $(y, Z)$ plane has a plate half gap $r_{p}$ and entrance corner radius $r_{c}$ as shown in Fig. 2 for the D1 lens. The plate potential is $V_{p}$ and maximum electric field is $E_{p}=V_{p} / r_{p}$. The resulting dimensionless defocusing field $d_{y 2}\left(r_{c} / r_{p}=10\right)$ at the entrance to the first lens D1 is shown in Fig. 4 and consists essentially of a single localized defocusing $\left(d_{y 2}>0\right)$ peak at $Z=45.6 \mathrm{~cm}$.

To calculate the resulting change in the $y$ velocity [Eq. (6)], we need the strengths of dimensionless defocusing powers $p_{y 2}, p_{y 4}, p_{y 6}$ which are plotted in Fig. 5 as a function of the corner radius ratio $r_{c} / r_{p}$. The D1-lens electrode has a corner radius $r_{c} / r_{p}=10$, for which the defocusing powers are $p_{y 2}=0.170, p_{y 4}=0.0125, p_{y 6}=$ 0.0023. Therefore an atom entering the D1 lens $\left(r_{p}=\right.$ $6 \mathrm{~mm}, r_{c}=60 \mathrm{~mm}, V_{p}=67.5 \mathrm{kV}$ ) with longitudinal velocity $V_{z i f}=3.0 \mathrm{~m} / \mathrm{s}$ and displacement $y=3 \mathrm{~mm}$ at the defocusing peak, will have its $y$ velocity increased by $\Delta v_{y i f}=110 \mathrm{~mm} / \mathrm{s}$ (or divergence $\Delta v_{y i f} / V_{z i f}=37 \mathrm{mr}$ ), with the nonlinear terms contributing only $\Delta v_{y i f}=$ $2 \mathrm{~mm} / \mathrm{s}$. This change in velocity is large compared with the atom bunch rms velocity $\sigma_{v_{y}}=8.0 \mathrm{~mm} / \mathrm{s}$ and we therefore have chosen the D1 lens to be $y$ focusing.

For the eEDM experiment itself, we only need a single dipole field lens (E4). If there was no end-field defocusing, then the maximum $\left(y, v_{y}\right)$ phase-space acceptance of the fountain is as shown in Fig. 6. This is fixed by the physical apertures in the $y$ direction, and for an E4 plate half gap of

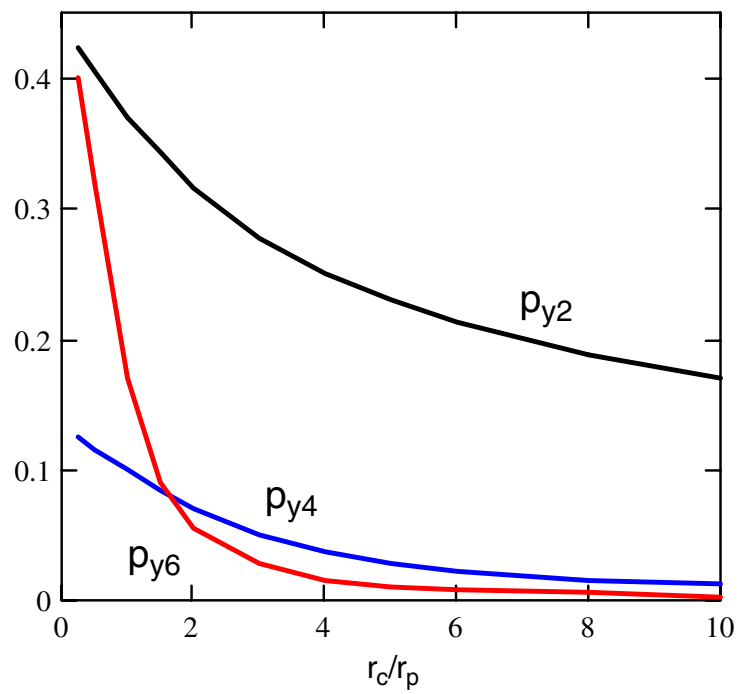

FIG. 5. The dimensionless defocusing powers $p_{y 2}, p_{y 4}, p_{y 6}$ [which determine the change in $y$ velocity: Eq. (6)] for the end field at the entrance of a lens (Fig. 4: D1 lens entrance), as a function of the electrode half gap $r_{p}$ and corner radius $r_{c}$. 


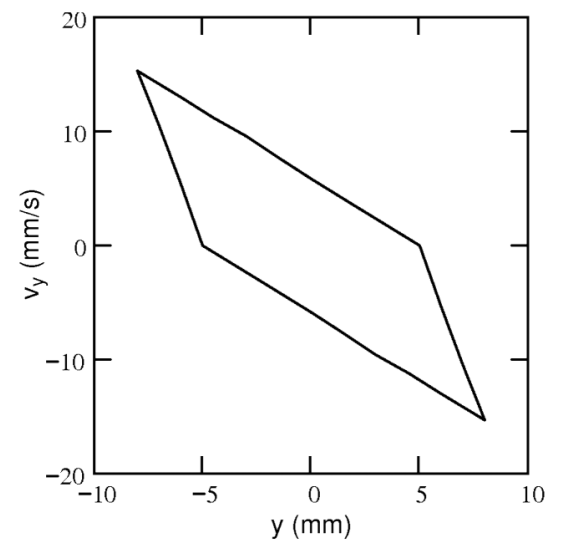

FIG. 6. The $\left(y, v_{y}\right)$ phase-space acceptance at the trap (area $A_{y}=153 \mathrm{~mm}^{2} / \mathrm{s}$ ), for the Cs fountain with only the E4 lens and the end-field defocusing at the entrance turned off.

$5 \mathrm{~mm}$ we obtain an acceptance area of $A_{y}=153 \mathrm{~mm}^{2} \mathrm{~s}^{-1}$. If the E4 half gap $r_{p}$ could be increased by $20 \%$ to $6 \mathrm{~mm}$, it would give a $44 \%$ larger phase-space acceptance area $\left[A_{y 2} / A_{y 2}=\left(r_{p 2} / r_{p 1}\right)^{2}\right]$.

With the defocusing end field turned on at the entrance of the E4 dipole field plates $\left(r_{p}=5 \mathrm{~mm}, r_{c}=50 \mathrm{~mm}\right.$, and $V_{p}=67.5 \mathrm{kV}$ ), the phase-space acceptance area is reduced to $A_{y}=0.0713 \mathrm{~mm}^{2} \mathrm{~s}^{-1}$, a factor of about 2000 less. In addition, the returning atom bunch is defocused in the $y$ direction to both a large size $(32 \mathrm{~mm})$ and large velocity spread $(130 \mathrm{~mm} / \mathrm{s})$.

So in order to compensate for the very large loss of the phase-space acceptance, as well as to focus the atom bunch, we need to add transverse-focusing lenses to the eEDM fountain.

\section{B. Gap field between lenses}

Between two lenses we have an electrode configuration as shown in Fig. 2 for the D1-F2, F2-D3, and D3-E4 lens gaps. The physical parameters are the lens gap length $L_{g}$, the plate half gaps $r_{p}$, and the corner radii $r_{c}, r_{d}$. The electrode potentials $V_{p}$ then give us the electric fields in each lens. In the eEDM fountain, because of the high fields and small lens gaps, all four lens pairs are set to the same plate potentials $V_{p}= \pm 67.5 \mathrm{kV}$.

In the eEDM fountain the D1-F2 and F2-D3 lens gaps have the same width $\left(L_{g}=1.5 \mathrm{~mm}\right)$, plate half gap $\left(r_{p}=\right.$ $6 \mathrm{~mm})$, and corner radii $\left(r_{c}=r_{d}=1.5 \mathrm{~mm}\right)$. Then as shown in Fig. 4, in each of the D1-F2 and F2-D3 lens gaps there are three $d_{y 2}$ peaks with a focusing peak in the center $\left(p_{y 2 F}=-0.0657\right)$ and two equal-strength defocusing peaks $\left(p_{y 2 D}=0.0343\right)$ on each side, resulting in a very small net defocusing $\left(p_{y 2 D F D}=0.0028\right)$ of the atoms.

We plot the linear-focusing/defocusing power $p_{y 2}$ in the $F / D$ peaks and their sum, in Fig. 7, as a function of the lens spacing $L_{g}\left(r_{c} / r_{p}=0.25\right)$. We see that as the lenses are

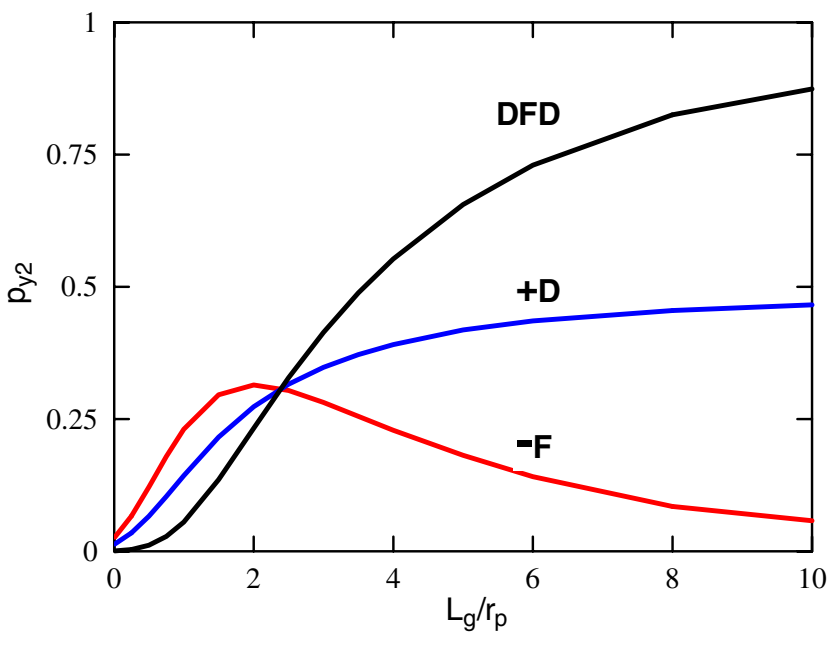

FIG. 7. The magnitude of the linear dimensionless focusing/ defocusing power $p_{y 2}$, for the end field between two lenses (Fig. 4: D1-F2 and F2-D3 gaps) with the same plate half gaps $r_{p}$ and corner radii $r_{c / d}=0.25 r_{p}$, as a function of the longitudinal lens spacing $L_{g}$. For the three impulse fields, $\mathrm{F}$ is for the central focusing peak, $\mathrm{D}$ is for each of the two defocusing peaks on each side, and DFD is their sum (2D-F).

moved apart the central focusing power $p_{y 2 F}$ first grows to reach a maximum around a few half gaps and then slowly goes to zero. The two defocusing peaks remain at the entrance/exit of the plates with the defocusing power $p_{y 2 D}$ growing as the electric field gradient increases with decreasing field between the lenses, and leveling off as the field goes to zero between the lenses-with a limiting value given by the single lens entrance/exit defocusing

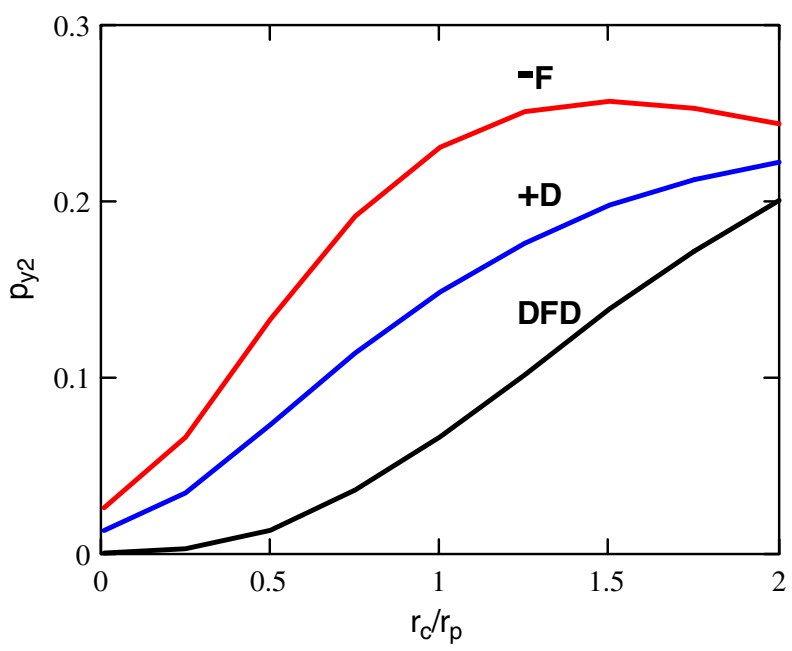

FIG. 8. The magnitude of the linear dimensionless focusing/ defocusing power $p_{y 2}$, for the end field between two lenses (Fig. 4: D1-F2 and F2-D3 gaps) with the same plate half gaps $r_{p}$ and longitudinal lens spacing $L_{g}=0.25 r_{p}$, as a function of the plate corner radius $r_{c}=r_{d}$. For the three impulse fields, $\mathrm{F}$ is for the central focusing peak, $\mathrm{D}$ is for each of the two defocusing peaks on each side, and DFD is their sum (2D-F). 
TABLE II. eEDM-lens end-field dimensionless defocusing power $p_{y 2}$.

\begin{tabular}{lcccccc}
\hline \hline & & \multicolumn{5}{c}{$p_{y 2}$} \\
D1-F2-D3 half gap & E4 half gap & D1 end & D1-F2 gap & F2-D2 gap & D2-E4 gap & Total sum \\
\hline $5 \mathrm{~mm}$ & $5 \mathrm{~mm}$ & 0.1698 & 0.0028 & 0.0028 & 0.0028 & 0.1782 \\
$6 \mathrm{~mm}$ & $5 \mathrm{~mm}$ & 0.0982 & 0.0016 & 0.0016 & 0.0308 & 0.1322 \\
\hline \hline
\end{tabular}

power calculated in the previous Sec. IVA. This plot shows how strongly the end-field defocusing is affected by neighboring lenses and their electric fields. This is very different from the case with charged particles for which the fringe fields of a focusing element (electric/magnetic quadrupole fields) are included in an effective length which is usually not affected by nearby elements.

To reduce the total defocusing in the gap between lenses, Fig. 7 shows us that the gap spacing between the lenses should be small. From Fig. 8, which plots the linearfocusing/defocusing power $p_{y 2}$ in the $F / D$ peaks and their sum for different corner radii $r_{c}\left(L_{g} / r_{p}=0.25\right)$, we see that to reduce the defocusing we also need small corner radii for the electrodes.

The large electric field of $13.5 \mathrm{MV} / \mathrm{m}$ for the eEDM experiment is needed only in the E4 dipole lens $\left(r_{p 4}=\right.$ $5 \mathrm{~mm}$ ). Therefore to further reduce the strong defocusing of the beam entering the D1 lens, we chose a larger plate half gap of $r_{p 1}=6 \mathrm{~mm}$ for the D1, F2, D3 lenses. Then in the D3-E4 lens gap $\left(r_{d 3}=1.5 \mathrm{~mm}, r_{c 4}=2.5 \mathrm{~mm}\right)$ as shown in Fig. 4, the $d_{y 2}$ field has a focusing peak $\left(p_{y 2 F}=-0.1072\right)$ followed by a single defocusing peak $\left(p_{y 2 D}=0.1378\right)$, resulting again in a small net defocusing $\left(p_{y 2 F D}=0.0306\right)$ of the atoms.

In Table II we compare the $p_{y 2}$ linear defocusing power in the four lens end-field regions shown in Fig. 4, for the case where the D1-F2-D3 triplet has the same plate half gap as E4 $(5 \mathrm{~mm})$, and the case with a larger triplet half gap (6 mm).

We see that, with a $6 \mathrm{~mm}$ plate half gap in the D1-F2-D3 lenses, the defocusing power in the D3-E4 gap increases by $\Delta p_{y}=0.028$ while in the other three end fields it decreases by $\Delta p_{y}=0.074$, resulting in an $26 \%$ reduction in the net defocusing power.

\section{TRANSVERSE-FOCUSING LENS FIELDS}

To compensate for the end-field defocusing in the lenses, as well as focus the atoms in both $x$ and $y$ directions, we need electrodes whose shape in the $(x, y)$ plane produces a transverse-focusing field. For charged particles an electrostatic lens with a pure quadrupole potential will give linear forces, with focusing in one direction and equal defocusing in the other direction. The total electric field of a quadrupole lens is $E^{2}=E_{A 2}^{2}\left(x^{2}+y^{2}\right)$, so for neutral atoms [Eq. (3)] it produces linear forces but they are defocusing in both $x$ and $y$ directions. The higher order pure multipole fields give nonlinear forces that are also defocusing in both directions. However, if a combination of a dipole and a sextupole potential is used, the resulting forces will focus the beam in one direction and defocus in the other $[2,5]$.

Inside the lenses, where the electrode is flat in the $z$ direction $(\partial \Phi / \partial Z=0)$, we choose the multipole potential $\Phi(x, y)$

$$
-\Phi(x, y)=E_{y o} y+E_{A 3}\left[x^{2} y-\frac{1}{3} y^{3}\right]
$$

where $E_{y o}$ is the electric dipole field, $E_{A 3} \equiv A_{3} E_{y o}$ is the sextupole field with relative strength $A_{3}$ whose sign gives the direction in which the atoms are focused:

$$
\begin{aligned}
& A_{3}<0 \text { then } F \text { lens ( } x \text { focus, } y \text { defocus) } \\
& A_{3}>0 \text { then } D \text { lens ( } x \text { defocus, } y \text { focus). }
\end{aligned}
$$

It also determines the transverse curvature $\Phi(x, y)=$ const of the electrode plates-the larger $A_{3}$ the greater is the curvature and the stronger the focusing.

The total electric field for this potential is

$E^{2}(x, y)=E_{y o}^{2}+2 E_{y o} E_{A 3}\left[x^{2}-y^{2}\right]+E_{A 3}^{2}\left[x^{2}+y^{2}\right]^{2}$,

where the first term is the field for a pure dipole and the third term for a pure sextupole potential. As we will see in the next section, the second term is the one that produces a linear force that focuses in one direction and defocuses equally in the other.

\section{ATOM TRAJECTORY EQUATIONS OF MOTION}

The total electric field of a set of lenses in a fountain that includes all the electric field terms considered in the previous two sections [Eqs. (4) and (7)] is

$$
\begin{aligned}
E^{2}(x, y, Z)= & E_{y o}^{2}(Z)+D_{y 2}(Z) y^{2}+\frac{1}{2} D_{y 4}(Z) y^{4} \\
& +\frac{1}{3} D_{y 6}(Z) y^{6}+2 E_{y o}(Z) E_{A 3}(Z)\left[x^{2}-y^{2}\right] \\
& +E_{A 3}^{2}(Z)\left[x^{2}+y^{2}\right]^{2},
\end{aligned}
$$

where $E_{y o}(Z) \equiv E(x=0, y=0, Z)$ is again the field on the optic axis.

A complete 3D modeling of the lenses is needed to obtain the $Z$ dependence of the sextupole field $E_{A 3}$ and also the strengths of possible additional electric field terms that are not included here and are not expected to be significant.

The longitudinal motion of the central trajectory $\left(x_{c}=0\right.$, $\left.v_{x c}=0, y_{c}=0, v_{y c}=0, Z_{c}, V_{z c}\right)$ can be calculated from 


$$
V_{z c} \frac{d V_{z c}}{d Z_{c}}=-g+\frac{\alpha}{m} E_{y o}\left(Z_{c}\right) E_{y 1}\left(Z_{c}\right) .
$$

This equation integrated gives us the conserved Hamiltonian [Eqs. (1) and (2)] from which we can calculate the magnitude of the central velocity $V_{z c}$ at any location in the fountain

$$
V_{z c}^{2}\left(Z_{c}\right)=V_{z c i}^{2}-2 g Z_{c}+\frac{\alpha}{m} E_{y o}^{2}\left(Z_{c}\right),
$$

where at the launch point $Z_{c}=0$ the velocity is $V_{z c}=V_{z c i}$ and the electric field $E_{y o}=0$.

In the generic fountain design optics considered here, the electric field and its gradient on axis $E_{y o}, E_{y 1}, E_{y 2}$ are shown in Fig. 3. The resulting change in the central velocity $V_{z c}$, from its initial launch velocity of $4 \mathrm{~m} / \mathrm{s}$, is plotted in Fig. 9. It shows that there is a significant increase in velocity entering the D1 and E4 lenses resulting in a much higher turnaround point of $1.10 \mathrm{~m}$ compared to $0.82 \mathrm{~m}$ with the lenses turned off.

For atoms launched along the optic axis $\left(x=0, v_{x}=0\right.$, $\left.y=0, v_{y}=0\right)$, the longitudinal equation of motion is

$$
\frac{d v_{z}}{d t}+G_{z} z=0
$$

with bunching field

$$
G_{z}\left(Z_{c}, z\right)=-\frac{\alpha}{m} D_{z e}\left(Z_{c}, z\right) z
$$

where

$$
D_{z e}\left(Z_{c}, z\right)=D_{z 2}\left(Z_{c}\right)+D_{z 3}\left(Z_{c}\right) z+D_{z 4}\left(Z_{c}\right) z^{2}+\cdots
$$

and

$$
\begin{gathered}
D_{z 2}=E_{y 1}^{2}+E_{y o} E_{y 2} \quad D_{z 3}=\frac{3}{2} E_{y 1} E_{y 2}+\frac{1}{2} E_{y o} E_{y 3} \\
D_{z 4}=\frac{1}{2} E_{y 2}^{2}+\frac{2}{3} E_{y 1} E_{y 3}+\frac{1}{6} E_{y o} E_{y 4} .
\end{gathered}
$$

For the eEDM fountain the linear dimensionless force field $d_{z 2} \equiv\left(r_{p}^{2} / E_{p}^{2}\right) D_{z 2}$ is plotted in Fig. 10, and consists of

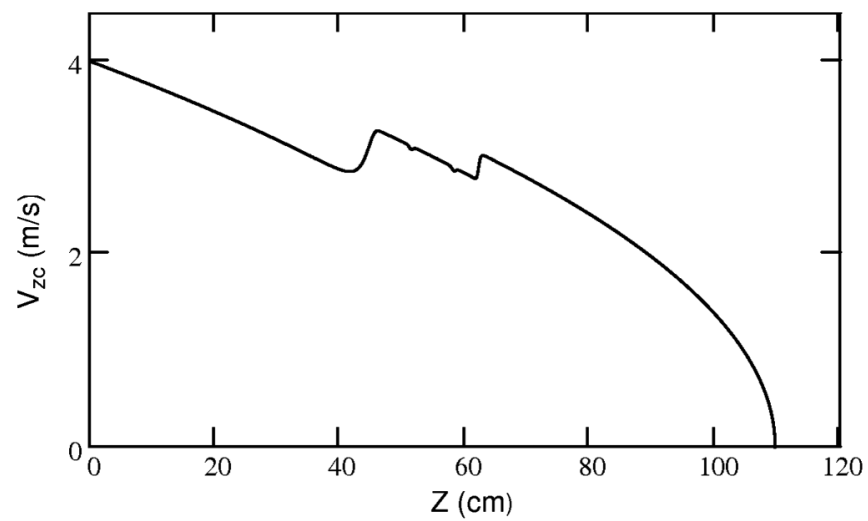

FIG. 9. The central longitudinal velocity $V_{z c}$ of the atom bunch in the Cs fountain, showing the electric end-field acceleration of the atoms on entering the D1 and E4 lenses (Fig. 3).

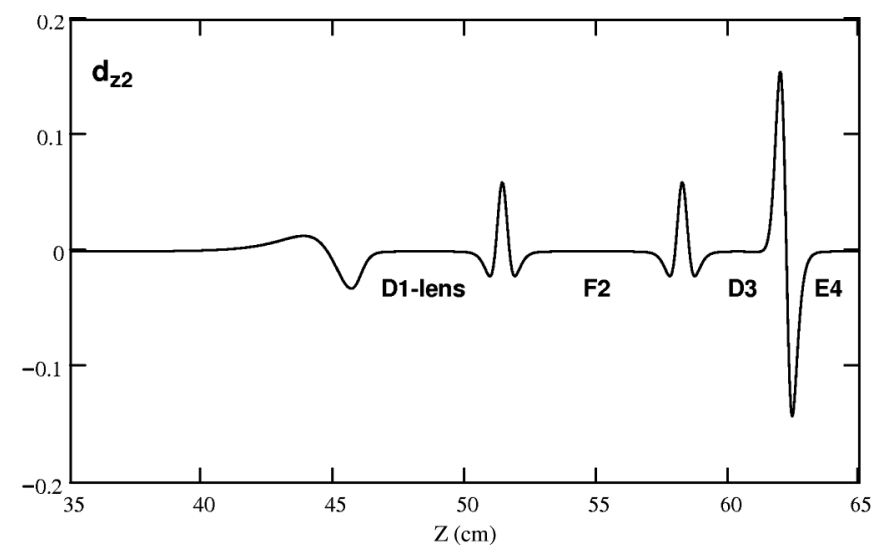

FIG. 10. The acceleration of the atom in the $z$ direction relative to the central trajectory is proportional to the dimensionless endfield bunching/debunching field $d_{z 2}$ (the linear term), and is shown here for the four lenses in the Cs fountain.

short-range bunching and debunching peaks at the lens ends and gaps, similar to that for the $d_{y 2}$ end-field defocusing function (Fig. 4).

In the transverse $(x, y)$ plane, the trajectory equations of motion are

$$
\frac{d v_{x}}{d t}+G_{x} x=0 \quad \frac{d v_{y}}{d t}+G_{y} y=0
$$

with focusing fields

$$
\begin{aligned}
G_{x}(x, y, Z)= & -\frac{\alpha}{m} 2 E_{A 3}(Z)\left[E_{y o}(Z)+E_{A 3}(Z)\left(x^{2}+y^{2}\right)\right] \\
G_{y}(x, y, Z)= & +\frac{\alpha}{m}\left\{2 E_{A 3}(Z)\left[E_{y o}(Z)-E_{A 3}(Z)\left(x^{2}+y^{2}\right)\right]\right. \\
& \left.-D_{y e}(y, Z)\right\}
\end{aligned}
$$

and longitudinal position $Z=Z_{c}+z$.

In the transverse $(x, y)$ plane the nonlinear force comes from the pure sextupole term in the total electric field [Eq. (8)].

To compare the focusing/defocusing power of a thin sextupole lens and the end field, we can integrate the $y$ velocity in the impulse approximation to get

$$
\Delta v_{y i f}=\frac{\alpha}{m} \frac{E_{p}^{2}}{V_{z i f}}\left(-2 A_{3} L_{\mathrm{eff}}+\frac{p_{y 2}}{r_{p}}\right) y,
$$

where $L_{\text {eff }}$ is the effective length of the sextupole lens. For the first D1 lens, and end-field defocusing peak $\left(p_{y e} / r_{p}=\right.$ $17 \mathrm{~m}^{-2}$ ) has $40 \%$ the strength of the sextupole focusing field $\left(2 A_{3} L_{\text {eff }}=42 \mathrm{~m}^{-2}\right)$.

For the eEDM fountain, with just the linear forces in the lenses, the $y$ direction phase-space acceptance at launch is shown in Fig. 11 (blue diamond) and has an area $A_{y}=$ $144 \mathrm{~mm}^{2} / \mathrm{s}$, which is $95 \%$ of the maximum geometric acceptance area (Fig. 6). This is a significant improvement over the $0.05 \%$ of the maximum acceptance we obtain using only the E4 dipole field plates (Sec. IVA). The 


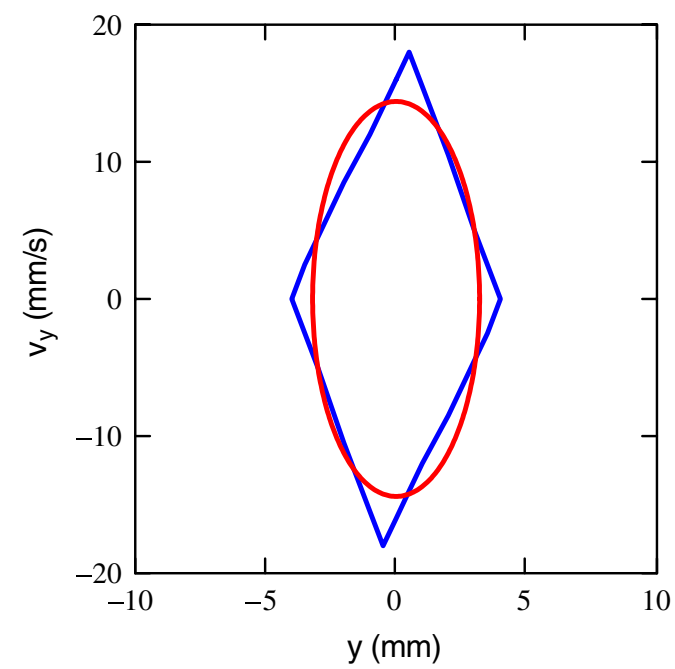

FIG. 11. The Cs fountain (Table I) $\left(y, v_{y}\right)$ phase-space acceptance area at the trap is shown in blue (area: $A_{y}=144 \mathrm{~mm}^{2} / \mathrm{s}$ ) for lens fields with only the linear-focusing terms. The initial phase-space ellipse used to calculate the atom bunch envelope $\sigma_{y}$ in Fig. 14 is shown in red.

boundary of the acceptance area shown is given by the trajectories, calculated from Eq. (10), that just graze the lens plates (Fig. 2).

With the nonlinear force terms turned on, the initial phase-space acceptance area (Fig. 11: blue) on returning to the launch position is shown in Fig. 12. We see that the nonlinear forces (red) distort the linear-force acceptance area (blue), with the larger velocity rays affected the most-but do not produce any significant decrease in acceptance.

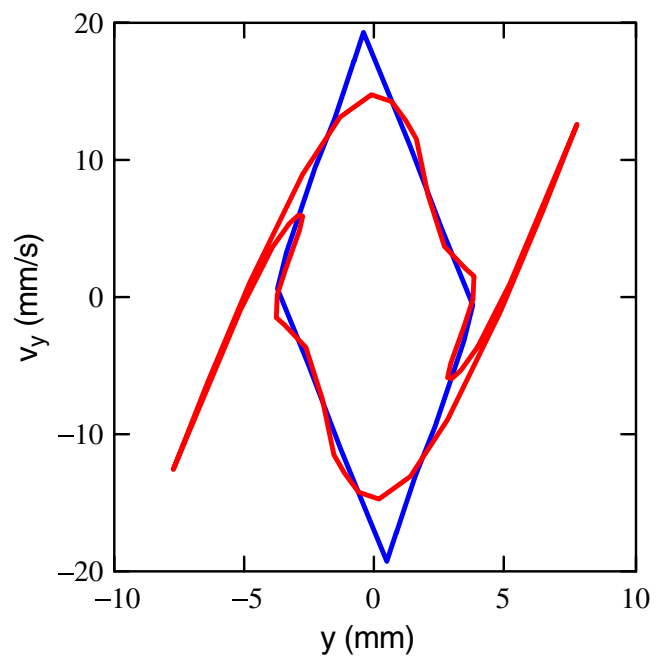

FIG. 12. The Cs fountain $\left(y, v_{y}\right)$ phase-space acceptance area (Fig. 11: blue) on returning to the trap is shown in blue for lens fields with only the linear-focusing terms, and in red with the nonlinear terms included.

\section{BEAM ENVELOPE EQUATIONS}

If the forces on the atom are linear and uncoupled, the focusing fields [Eqs. (9) and (10)] become

$$
\begin{aligned}
G_{x}\left(Z_{c}\right) & =-\frac{\alpha}{m} 2 E_{A 3}\left(Z_{c}\right) E_{y o}\left(Z_{c}\right) \\
G_{y}\left(Z_{c}\right) & =+\frac{\alpha}{m}\left[2 E_{A 3}\left(Z_{c}\right) E_{y o}\left(Z_{c}\right)-D_{y 2}\left(Z_{c}\right)\right] \\
G_{z}\left(Z_{c}\right) & =+\frac{\alpha}{m} D_{z 2}\left(Z_{c}\right)
\end{aligned}
$$

and they depend only on the central trajectory position $Z_{c}$.

Then, for any beam distribution in phase space, we can calculate the evolution of the root-mean-square (rms) moments $\sigma_{x}^{2}=\left\langle x^{2}\right\rangle, \sigma_{x v_{x}}=\left\langle x v_{x}\right\rangle, \sigma_{v_{x}}^{2}=\left\langle v_{x}^{2}\right\rangle$, etc., from the envelope equations [12] for the $x$ direction (focusing if $G_{x}>0$, and defocusing for $G_{x}<0$ )

$$
\begin{gathered}
\frac{d \sigma_{x}^{2}}{d t}-2 \sigma_{x v_{x}}=0 \quad \frac{d \sigma_{x v_{x}}}{d t}-\sigma_{v_{x}}^{2}+G_{x}\left(Z_{c}\right) \sigma_{x}^{2}=0 \\
\frac{d \sigma_{v_{x}}^{2}}{d t}+2 G_{x}\left(Z_{c}\right) \sigma_{x v_{x}}=0
\end{gathered}
$$

and equations of the same form for the $y$ and $z$ directions. Here at time $t$ the central atom is at the longitudinal position $Z_{c}$ with velocity $V_{z c}$.

The above three envelope equations are not independent, but are connected by the constant of the motion the rms emittance: $\varepsilon_{x}=\sqrt{\sigma_{x}^{2} \sigma_{v_{x}}^{2}-\sigma_{x v_{x}}^{2}}$.

At any position in the fountain, the atom trajectories of the rms distribution lie inside the equivalent KapchinskyVladimirsky distribution $[13,14]\left(x, v_{x}\right)$ phase-space ellipse given by

$$
\sigma_{v_{x}}^{2} x^{2}-2 \sigma_{x v_{x}} x v_{x}+\sigma_{x}^{2} v_{x}^{2}=\varepsilon_{x}^{2}
$$

with area $A_{x}=\pi \varepsilon_{x}$. At a waist $\left(\sigma_{x v_{x}}=0\right)$, such as the fountain launch point, the phase-space ellipse is upright with $\left(x^{2} / \sigma_{x}^{2}\right)+\left(v_{x}^{2} / \sigma_{v_{x}}^{2}\right)=1$ and $\varepsilon_{x}=\sigma_{x} \sigma_{v_{x}}$.

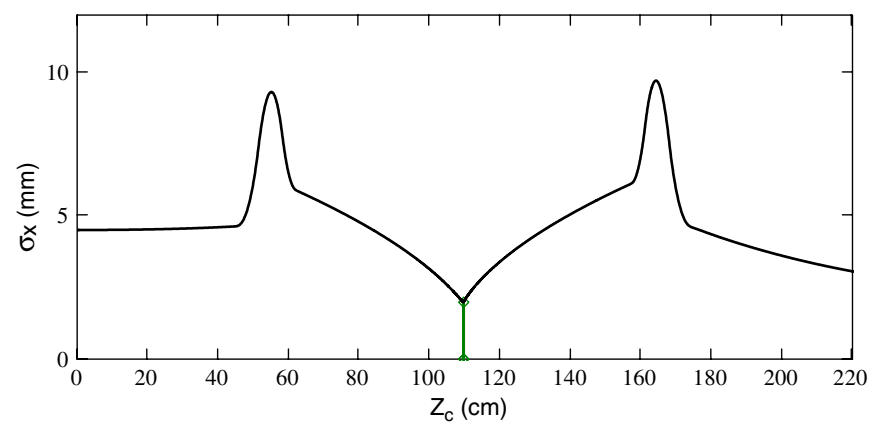

FIG. 13. The atom bunch $\sigma_{x}$ envelope in the Cs fountain, for launch velocity $V_{z c}=4.0 \mathrm{~m} / \mathrm{s}$ and an initial phase-space ellipse at the trap with beam size $\sigma_{x}=4.5 \mathrm{~mm}$, tilt $\sigma_{x v_{x}}=0$, velocity $\sigma_{v_{x}}=8.0 \mathrm{~mm} / \mathrm{s}$, and area $A_{x}=113 \mathrm{~mm}^{2} / \mathrm{s}$. The green line marks the turnaround point at $110 \mathrm{~cm}$. 


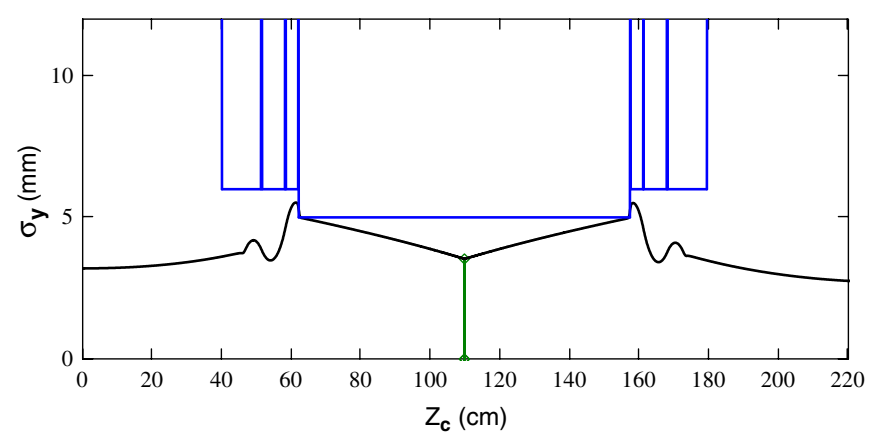

FIG. 14. The atom bunch $\sigma_{y}$ envelope in the Cs fountain, for launch velocity $V_{z c}=4.0 \mathrm{~m} / \mathrm{s}$ and an initial phase-space ellipse at the trap with beam size $\sigma_{y}=3.2 \mathrm{~mm}$, tilt $\sigma_{y v_{y}}=0$, velocity $\sigma_{v_{y}}=14.4 \mathrm{~mm} / \mathrm{s}$, and area $A_{y}=145 \mathrm{~mm}^{2} / \mathrm{s}$. The green line marks the turnaround point at $110 \mathrm{~cm}$, and blue shows the electrode apertures in the four lenses.

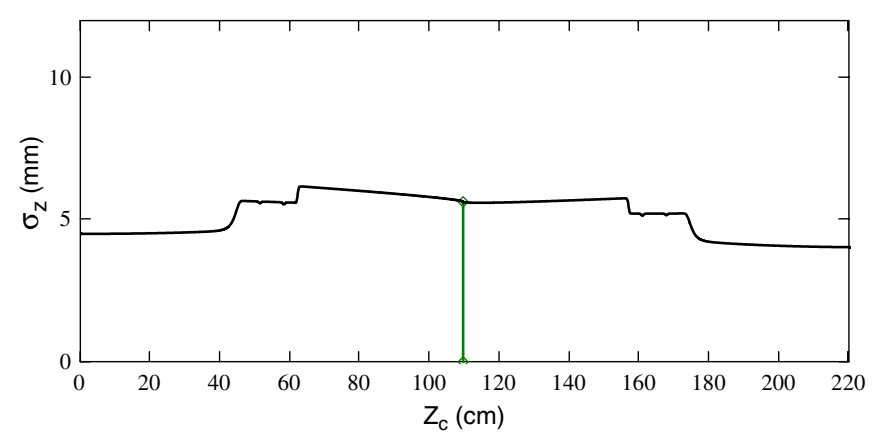

FIG. 15. The atom bunch $\sigma_{z}$ envelope in the Cs fountain, for launch velocity $V_{z c}=4.0 \mathrm{~m} / \mathrm{s}$ and an initial phase-space ellipse at the trap with beam size $\sigma_{z}=4.5 \mathrm{~mm}$, tilt $\sigma_{z v_{z}}=0$, velocity $\sigma_{v_{z}}=8.0 \mathrm{~mm} / \mathrm{s}$, and area $A_{z}=113 \mathrm{~mm}^{2} / \mathrm{s}$. The green line marks the turnaround point at $110 \mathrm{~cm}$.

For the generic eEDM fountain with lens parameters given in Table I, we show in Fig. 13 the beam envelope size $\sigma_{x}$ for an initial phase-space ellipse with rms sizes $\sigma_{x}=$ $4.5 \mathrm{~mm}, \sigma_{x v_{x}}=0, \sigma_{v_{x}}=8.0 \mathrm{~mm} / \mathrm{s}, A_{x}=113 \mathrm{~mm}^{2} / \mathrm{s}$. The actual acceptance in the $x$ direction will be determined by the fountain apertures and the good-field width of the lenses.

The beam envelope size $\sigma_{y}$, which is limited by the plate half gaps, is shown in Fig. 14 for an initial phase-space ellipse with $\sigma_{y}=3.2 \mathrm{~mm}, \sigma_{y v_{y}}=0, \sigma_{v_{y}}=14.4 \mathrm{~mm} / \mathrm{s}$, $A_{y}=145 \mathrm{~mm}^{2} / \mathrm{s}$, matched approximately to the trajectory acceptance area shown in Fig. 11.

The beam longitudinal envelope size $\sigma_{z}$ for an initial phase-space ellipse with rms sizes $\sigma_{z}=4.5 \mathrm{~mm}$, $\sigma_{z v_{z}}=0, \sigma_{v_{z}}=8.0 \mathrm{~mm} / \mathrm{s}, A_{z}=113 \mathrm{~mm}^{2} / \mathrm{s}$ is shown in Fig. 15, and we find that the atoms remain well bunched in the fountain. Part of this comes from the conservation of the Hamiltonian energy, which shows us that for any two atoms on the optic axis, the relative kinetic energy at all positions $Z$ is constant, i.e., $\frac{1}{2}\left(V_{z}^{2}-V_{z c}^{2}\right)=\left(V_{z c}+\frac{1}{2} v_{z}\right) v_{z}=$ const, and is the same as that at the launch point.

\section{CONCLUSIONS}

In experiments where a neutral atom bunch transits through electrostatic lenses, the defocusing of the atoms in the end fields of the lenses can be significant if the atoms are very slow and the lenses have high fields and small plate gaps. We have described in this paper the characteristics of these defocusing end fields, and how they can be overcome by the introduction of transverse-focusing lenses to keep the atom bunch small and maximize the transmission. These results apply to electric fields of any strength and can be used in other applications such as atomic clocks and for transport and focusing on any strong-field-seeking polar molecule.

\section{ACKNOWLEDGMENTS}

The author thanks Harvey Gould, Ben Feinberg, David Kilcoyne, Charles Munger, and Hiroshi Nishimura for their help in understanding the experimental needs and the atom beam optics in the eEDM fountain. This work was supported by the Director, Office of Science, of the U.S. Department of Energy under Contract No. DE-AC02$05 \mathrm{CH} 11231$.

[1] H.-R Noh, K. Shimizu, and F. Shimizu, Phys. Rev. A 61, 41601 (2000).

[2] J. G. Kalnins, G. Lambertson, and H. Gould, Rev. Sci. Instrum. 73, 2557 (2002).

[3] H. Nishimura, G. Lambertson, J.G. Kalnins, and H. Gould, Rev. Sci. Instrum. 74, 3271 (2003).

[4] H. Nishimura, G. Lambertson, J.G. Kalnins, and H. Gould, Eur. Phys. J. D 31, 359 (2004).

[5] J. G. Kalnins, J. M. Amini, and H. Gould, Phys. Rev. A 72, 043406 (2005).

[6] H.L. Bethlem, M.R. Tarbutt, J. Kupper, D. Carty, K. Wohlfart, E. A. Hinds, and G. Meijer, J. Phys. B 39, R263 (2006).

[7] E. Simon, P. Laurent, and A. Clairon, Phys. Rev. A 57, 436 (1998).

[8] J. M. Amini and H. Gould, Phys. Rev. Lett. 91, 153001 (2003).

[9] J. M. Amini, C. T. Munger, and H. Gould, Phys. Rev. A 75, 063416 (2007).

[10] K. Gibble, S. Chang, and R. Legere, Phys. Rev. Lett. 75, 2666 (1995).

[11] R. Legere and K. Gibble, Phys. Rev. Lett. 81, 5780 (1998).

[12] F. J. Sacherer, IEEE Trans. Nucl. Sci. 18, 1105 (1971).

[13] M. Reiser, Theory and Design of Charged Particle Beams (Wiley, New York, 1994), p. 358.

[14] I. M. Kapchinsky and V. V. Vladimirsky, in Proceedings of the International Conference on High Energy Accelerators (CERN, Geneva 1959), p. 274. 